\title{
共价有机框架新应用：选择性水解二维共价有机框架制备有机纳米管
}

\author{
王 为* \\ (兰州大学化学化工学院 功能有机分子化学国家重点实验室 兰州 730000)
}

\section{New Application of Covalent Organic Frameworks (COFs): Fabricating Organic Nanotubes through Selective Hydrolysis of Two-Dimensional COFs}

\author{
Wang, Wei* \\ (State Key Laboratory of Applied Organic Chemistry, College of Chemistry and Chemical Engineering, \\ Lanzhou University, Lanzhou 730000)
}

共价有机框架(covalent organic frameworks, $\mathrm{COFs}$ ) 是一类晶态有机多孔聚合物, 它们通过多官能团有机单 体分子缩聚形成共价键连接的二维或三维拓展网格结 构 ${ }^{[1]}$. 遵循 “框架化学” 构筑原理 ${ }^{[2]}, \mathrm{COFs}$ 的结构可被 预先设计并精确构筑. 这类新颖材料的显著特点是其内 部分布高度有序的纳米孔道且孔道形状和大小可通过 改变聚合单体的对称性和尺寸进行精确调节. 此外, 从 构效关系的角度考虑, 多孔和共轭结构特征 ${ }^{[3]}$ 使其在物 质吸附、储存与分离、催化、光电和传感检测等领域均 得到了引人注目的应用 ${ }^{[4]}$. 尽管如此, 开辟 $\mathrm{COFs}$ 新应 用的需求仍然十分迫切.

中国科学院上海有机化学研究所赵新课题组一直 致力于新结构晶态有机多孔聚合物的研究工作. 2014 年 以来他们 ${ }^{[5 \sim 9]}$ 开发出了一种新类型的二维 $\mathrm{COFs}$ 一一异 孔共价有机框架(异孔 COFs). 与绝大多数二维 $\mathrm{COFs}$ 具 有均一孔结构不同, 在异孔 COFs 中, 每一种 COF 同时 具有不同种类(两种或以上)的孔结构, 从而赋予这类 $\mathrm{COFs}$ 高度有序的等级孔, 可表现出与均一孔 COFs 不一 样的性质、功能与应用. 最近, 赵新课题组 ${ }^{[10]}$ 充分利用 异孔 COFs 的结构特点与优势, 结合对二维 COFs 结构 的新认识, 成功实现了通过选择性解体二维 COFs 来可 控制备有机纳米管, 从而发展出 COFs 的一种全新应用. 纳米管是一类管状纳米材料, 具有独特结构与性质, 自 1991 年碳纳米管被发现以来就备受关注, 目前主要通 过原子/分子聚合或组装等 “自下而上” 的方法来获得, 其可控制备还面临很多挑战.
基于二维 $\mathrm{COFs}$ 具有垂直于层间堆积方向和平行有 序排列的一维孔道这一特点, 他们从新的角度去理解二 维 $\mathrm{COFs}$, 将这些孔道视为平行有序排列的 “纳米管阵 列”. 因此, 如果能够选择性地断裂孔道间的连接点, 就 有望解体 “纳米管阵列” 获得分散的纳米管, 从而实现 “自上而下”制备纳米管, 并通过调控 COFs 结构来实现 对纳米管结构(形状及孔径)的精确调控. 然而, 均一孔 COFs 由于具有全同的孔道结构, 无法选择性断裂特定 部位. 与之相比, 异孔 COFs 具有不同结构/性质的等级 孔, 为选择性定点解离提供了基础. 基于这一思路, 他 们设计合成了利用硼氧键和腙键两种动态共价键正交 反应缩聚而成的双孔 COF 材料(COF-OEt, 图 1), 该 $\mathrm{COF}$ 中一种孔道由腙键连接单体基元而形成, 另外一 种孔道则是由硼氧六环连接前者而成, 其结构通过多种 光谱、 $X$ 射线粉末衍射(PXRD)以及氮气吸附实验证实. 得到目标异孔 COF 后, 他们将其分散在酸性水溶液中, 在此条件下硼氧键被水解而腙键不受影响. 水解产物经 透射电子显微镜(TEM)表征, 与前体 COF 具有层状结构 不同, 水解后层状结构消失, 清晰地观察到管状结构, 证实获得了预期的纳米管(图 $2 a$ 和 $2 b$ ).

为了进一步提升所得有机纳米管的稳定性, 他们还 设计合成了带有烯丙基侧链的类似结构双孔 COF, 通过 烯烃复分解反应对其进行层间交联, 再进行水解断裂硼 氧键得到纳米管(NT-OAl-CR), 研究发现超声后这些纳 米管仍然可稳定存在，表明层间交联极大地提高了纳米 管的稳定性(图 2c 和 2d).

* Corresponding author. E-mail: wang_wei@lzu.edu.cn. Published online February 9, 2020. 


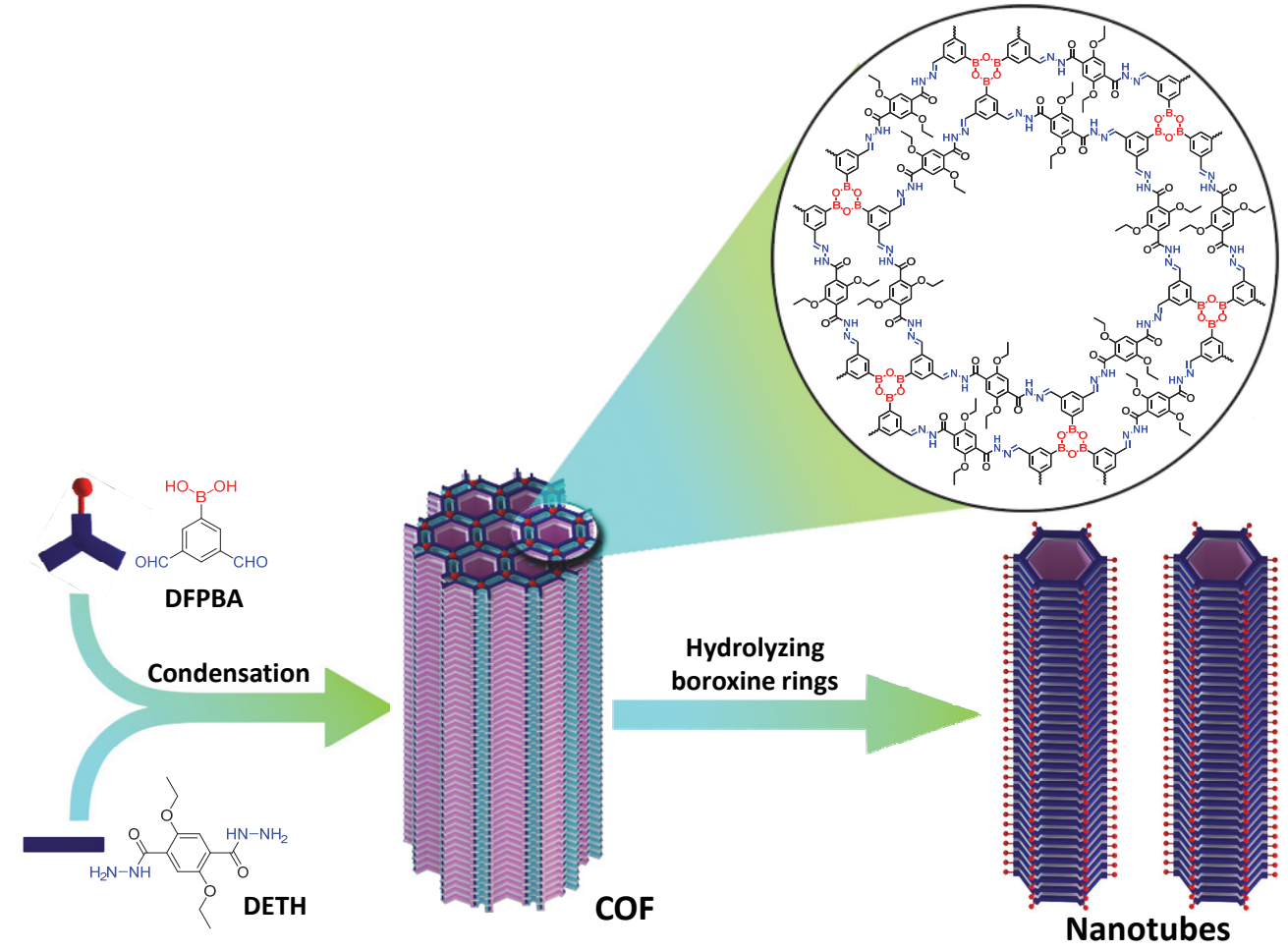

图 1 选择性水解二维异孔 COF 制备有机纳米管示意图

Figure 1 Schematic illustration for fabrication of nanotubes through selectively hydrolyzing a 2D heteropore COF
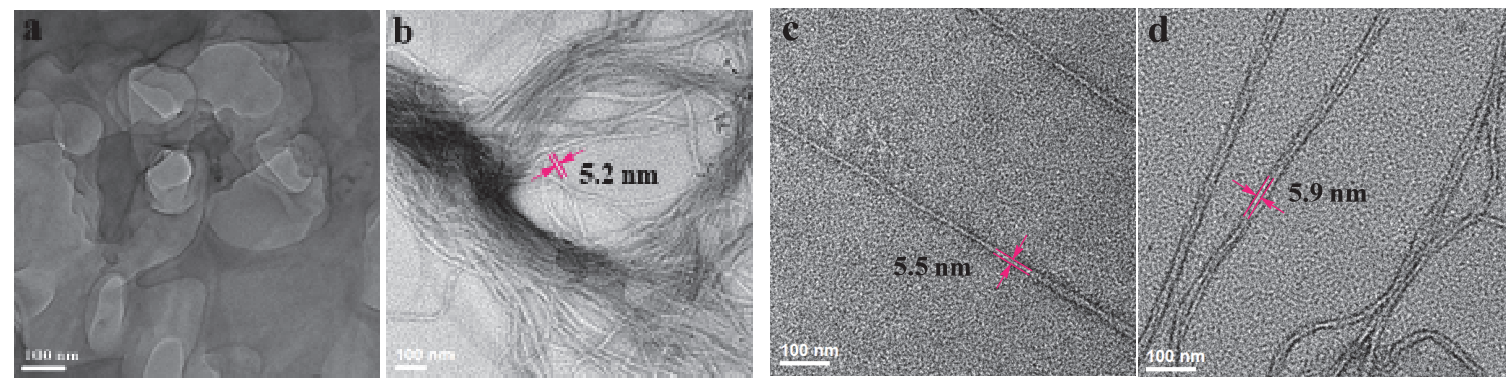

图 2 COF-OEt 水解前(a)及水解后(b)以及 NT-OAl-CR 超声前(c)及超声后(d)的 TEM 图

Figure 2 TEM images of COF-OEt before (a) and after (b) hydrolysis, and NT-OAl-CR before (c) and after (d) ultrasonic treatment

总之, 赵新课题组基于对二维 COFs 结构新的理解, 发展了选择性水解二维 COFs 可控制备有机纳米管的方 法, 为 “自上而下” 制备纳米管提供了新思路. 该研究 开发了 COFs一种全新的应用, 并首次展示了异孔 COFs 不同于均一孔 COFs 的独特性质与应用.

\section{References}

[1] Ding, S.-Y.; Wang, W. Chem. Soc. Rev. 2013, 42, 548.

[2] Rungtaweevoranit, B.; Diercks, C. S.; Kalmutzki, M. J.; Yaghi, O. M. Faraday Discuss. 2017, 201, 9.

[3] Côté, A. P.; Benin, A. I.; Ockwig, N. W.; O’Keeffe, M.; Matzger, A.
J.; Yaghi, O. M. Science 2005, 310, 1166.

[4] Song, Y.; Sun, Q.; Aguila, B.; Ma, S. Adv. Sci. 2019, 6, 1801410.

[5] Zhou, T.-Y.; Xu, S.-Q.; Wen, Q.; Pang, Z.-F.; Zhao, X. J. Am. Chem. Soc. 2014, 136, 15885.

[6] Pang, Z.-F.; Xu, S.-Q.; Zhou, T.-Y.; Liang, R.-R.; Zhan, T.-G.; Zhao, X. J. Am. Chem. Soc. 2016, 138, 4710.

[7] Qian, C.; Qi, Q.-Y.; Jiang, G.-F.; Cui, F.-Z.; Tian, Y.; Zhao, X. J. Am. Chem. Soc. 2017, 139, 6736.

[8] Liang, R.-R.; Xu, S.-Q.; Zhang, L.; A, R.-H.; Chen, P.; Cui, F.-Z.; Qi, Q.-Y.; Sun, J.; Zhao, X. Nat. Commun. 2019, 10, 4609.

[9] Liang, R.-R.; Zhao, X. Org. Chem. Front. 2018, 5, 3341.

[10] Liang, R.-R.; A, R.-H.; Xu, S.-Q.; Qi, Q.-Y.; Zhao, X. J. Am. Chem. Soc. 2020, 142, 70 .

(Zhao, C.) 